\title{
Avaliação da efetividade da assistência pré-natal de uma Unidade de Saúde da Família em um município da Grande São Paulo
}

\author{
Evaluation of effectivenness of prenatal assistance in a health Family Unit in a city of the São Paulo State \\ Evaluación de la efectividad de la asistencia prenatal de una Unidad de Salud de la Familia en un municipio del Estado \\ de São Paulo
}

\author{
Roselane Gonçalves', Maristela Belletti Mutt Urasaki", \\ Miriam Aparecida Barbosa Merighi'", Carla Gisele D'AvilaIV \\ 'Universidade de São Paulo. Escola de Artes, Ciências e Humanidades. São Paulo, SP \\ "Universidade de Mogi das Cruzes. Mogi das Cruzes, SP \\ "'Universidade de São Paulo. Escola de Enfermagem. Departamento Materno-Infantil e PsiQuiátrica. São Paulo, SP \\ "Nrograma de Saúde da Família. Suzano, SP
}

Submissão: 13/10/2007

Aprovação: $21 / 01 / 2008$

\section{RESUMO}

Dada a importância da Qualidade do cuidado pré-natal na redução da morbi-mortalidade materna e perinatal, este estudo objetivou avaliar a Qualidade da assistência prestada à gestantes de baixo risco por meio dos indicadores de Qualidade preconizados pelo Ministério da Saúde. Trata-se de um estudo avaliativo, Quantitativo e retrospectivo realizado em um Serviço de Pré-Natal de uma Unidade de Saúde da Família de um Município da Grande São Paulo. Os resultados evidenciaram que o Programa de Saúde da Família propicia assistência pré-natal de Qualidade, e Que o vínculo estabelecido entre os profissionais da Unidade e os Agentes Comunitários de Saúde com as gestantes é imprescindível para a adesão das mesmas ao Programa de Assistência Pré-Natal.

Descritores: Assistência pré-natal; Saúde da mulher; Programa saúde da família.

\begin{abstract}
Considering the importance of prenatal care Quality to reduce maternal and perinatal morbidity and mortality, the aim of this study was to evaluate Quality in the assistance to low-risk pregnant women by means of Quality indicators stated by the Health Ministry. This is an evaluating, Quantitative and retrospective study carried out in a Prenatal Service in a Family Health Unit in a city in the state of São Paulo. The results showed that the Family Health Program provides a Quality prenatal assistance and that the link established between the Unit professionals and the Health Community Agents with pregnant women is imperative for the adherence to the Prenatal Assistance Program.
\end{abstract}

Descriptors: Prenatal assistance; Women's health; Family health program.

\section{RESUMEN}

Visto la importancia de la calidad del cuidado prenatal en la reducción de la morbidad y mortalidad materna y perinatal, este estudio tuvo como objetivo evaluar la calidad de la asistencia prestada a las mujeres embarazadas de bajo riesgo por medio de los indicadores de calidad preconizados por el Ministerio de la salud. Este es un estudio evaluativo, cuantitativo y retrospectivo realizado en un Servicio Prenatal de una Unidad de Salud de la Familia de un Municipio del Estado de São Paulo. Los resultados mostraron Que el Programa de Salud de la Familia propicia asistencia prenatal de calidad y que el vínculo establecido entre los profesionales de la Unidad y los Agentes Comunitarios de la salud con las mujeres embarazadas es imprescindible para la adhesión de las mismas al programa de Asistencia Prenatal.

Descriptores: Asistencia prenatal; Salud de la mujer; Programa de salud de la familia.

Correspondência: Miriam Aparecida Barbosa Merighi. Universidade de São Paulo - Escola de Enfermagem. Rua Enéas de Carvalho Aguiar, 419. CEP 05403-000. São Paulo, SP. 


\section{INTRODUÇÃO}

A cobertura do pré-natal consiste num dos principais indicadores do Pacto da Atenção Básica do SUS, sendo Que a assistência prestada envolve toda a cadeia de procedimentos Que os serviços devem realizar para outras ações de atenção básica. Desta forma, o seu funcionamento reflete aspectos da atuação de outros programas e a sua importância é evidente expressando-se no conjunto de normas Que regem a operacionalização do SUS. O impacto sobre a prevenção da prematuridade e do baixo peso ao nascer tem sido amplamente documentado ${ }^{(1)}$.

A assistência à gestante, uma das atividades realizadas há tempo nos serviços públicos de saúde no país, foi, por muitos anos, orientada principalmente para melhorar os indicadores da saúde infantil. No entanto, um novo paradigma na atenção à saúde da mulher foi concebido pelo movimento de mulheres que, associado às discussões técnicas promovidas pelos profissionais de saúde, culminou nas bases programáticas do Programa de Assistência Integral à Saúde da Mulher (PAISM), instituído pelo Ministério da Saúde em 1983.

O PAISM, na sua forma mais abrangente preconiza assistir às necessidades globais de saúde da mulher, nos aspectos clinicoginecológicos e educativos, voltados ao aperfeiçoamento do controle pré-natal, do parto e puerpério; direciona a abordagem dos problemas presentes desde a adolescência até a terceira idade; busca o controle das doenças transmitidas sexualmente, do câncer cérvico-uterino e mamário e abrange a assistência para concepção e contracepção $0^{(2)}$.

No Que se refere a assistência pré-natal, o PAISM estabelece um conjunto de procedimentos clínicos e educativos com o objetivo de promover a saúde e identificar precocemente os problemas Que possam resultar em risco para saúde da gestante e do concepto ${ }^{(2,3)}$. Entendendo-se como risco toda a característica ou circunstância Que está associada ao aumento de probabilidade de ocorrência de um fato indesejado necessariamente em sua causalidade. Considerase de alto risco ou de risco agravado a prenhez em Que a gestante ou o seu concepto está sujeito a sucumbir ou ser lesado durante o ciclo grávido-puerperal $\left.\right|^{(4,5)}$.

A assistência pré-natal tem ainda o objetivo de orientar e esclarecer sobre o parto e os cuidados com o recém-nascido, visando a redução das taxas de morbi-mortalidade materno-infantil, baixo peso ao nascer e retardo do crescimento intra-uterino, visto Que estas causas são evitáveis dependendo da Qualidade assistencial prestada neste período $0^{(4,6)}$

O pré-natal deve ser organizado para atender às reais necessidades da população de gestantes por meio da utilização de conhecimentos técnico-científicos e recursos adequados e disponíveis para cada caso. Reforça-se, ainda, Que as ações de saúde precisam estar voltadas para cobertura de toda a população alvo da área de abrangência da unidade de saúde, assegurando a continuidade no atendimento, o acompanhamento e a avaliação dessas ações sobre a saúde materna-perinatal ${ }^{(7)}$.

Neste sentido, visando garantir, entre outras, a identificação precoce de todas as gestantes da comunidade e o pronto início do acompanhamento no primeiro trimestre da gravidez, bem como a operacionalização do sistema de referência e contra-referência, foram estabelecidas condições para uma assistência pré-natal efetiva objetivando garantir a continuidade da assistência em todos os níveis de complexidade do sistema de saúde ${ }^{(7)}$.

Vale ressaltar Que a avaliação da Qualidade do pré-natal permanente permite identificar problemas de saúde da população alvo, e monitora o desempenho do serviço. Os resultados obtidos de tal avaliação poderão subsidiar tanto a manutenção das estratégias Quanto a sua modificação, com vistas na melhor Qualidade da assistência ${ }^{(8)}$.

Em contrapartida, alguns autores afirmam Que os estudos sobre a extensão dos cuidados são importantes, mas não fornecem informações sobre o conteúdo, a continuidade e a Qualidade da assistência prestada. Lembrando Que a Qualidade da assistência prestada pelo serviço e pelos profissionais de saúde depende da adesão das mulheres ao programa de pré-natal e esta adesão é essencial para redução dos elevados índices de mortalidade materna e perinatal, uma vez que é do nosso conhecimento Que $98 \%$ das mortes das mulheres por causas maternas são evitáveis mediante a adoção de medidas relativamente simples, tais como melhorar a Qualidade do cuidado perinatal e garantir o acesso ao serviço de saúde. Vale salientar Que outras características sócio-ecônomicas, culturais e demográficas tem um potencial maior para avaliar o acesso e a Qualidade dos serviços ${ }^{(7,8)}$.

Estudos apontam Que, nos paises subdesenvolvidos, onde a assistência médica é precária, a atenção pré-natal representa a única oportunidade para as mulheres receberem assistência médica ${ }^{(2,7)}$. Entretanto, ainda hoje, a cobertura da assistência pré-natal no Brasil ainda é baixa, apesar de vir aumentando nas últimas décadas ${ }^{(7,8)}$.

Vários autores comentam, Que apesar da assistência pré-natal ter estado sempre presente no escopo das ações praticadas pelos serviços de saúde, até o momento, permanecem Questões Que, entre outras, devem ser discutidas, como o acesso em algumas regiões e áreas do país, a Qualidade da atenção prestada, o vínculo entre o pré-natal e o parto, a humanização da atenção e as inaceitáveis taxas de mortalidade materna e perinatal ${ }^{(7,9-11)}$.

Diante da necessidade de garantir atenção pré-natal de Qualidade e de reduzir as altas taxas de morbi-mortalidade materna e perinatal, o Ministério da Saúde elaborou no ano de 2000 o Programa de Humanização no Pré-Natal e Nascimento (PHPN), cuja pretensão é ser um marcador de águas anunciando o paradigma da humanização como novo modelo de atenção à mulher durante a gestação e o parto. O PHPN propõe indicadores de desempenho e Qualidade da assistência pré-natal, além de disponibilizar incentivos financeiros aos municípios Que adQuirirem este programa ${ }^{(12,13)}$. Suas principais estratégicas são: assegurar a melhoria do acesso, da cobertura e da Qualidade do acompanhamento pré-natal, assistência ao parto e puerpério, na perspectiva dos direitos de cidadania. O Programa fundamenta-se no direito à humanização da assistência obstétrica e neonatal como condição primeira para o adequado acompanhamento do parto e puerpério ${ }^{(12)}$.

A humanização compreende, entre outros, dois aspectos fundamentais. O primeiro, diz respeito à convicção de Que é dever das unidades de saúde receber com dignidade a mulher, seus familiares e o recém-nascido. Isto reQuer a adoção de atitude ética e solidária por parte dos profissionais de saúde, organização da instituição, de modo a criar um ambiente acolhedor, e a implementação de condutas hospitalares Que rompam com o tradicional isolamento imposto à mulher durante o processo de parturição. O segundo, refere-se à adoção de medidas e 
procedimentos sabidamente benéficos para o acompanhamento do parto e do nascimento, evitando práticas intervencionistas desnecessárias Que, embora tradicionalmente realizadas, não beneficiam a mulher nem o recém-nascido e Que, com freqüência, acarretam maiores riscos para ambos ${ }^{(12)}$.

Para garantir a Qualidade da assistência pré-natal conforme a filosofia do PHPN o Ministério da Saúde estabeleceu diretrizes para atenção pré-natal, dentre elas figuram desde rotinas preconizadas para consultas até a definição de fatores de risco na gravidez, de forma que se possa ampliar a assistência pré-natal incluindo, entre outras normas, a participação do enfermeiro como membro da equipe de saúde Que presta assistência direta à mulher durante o ciclo gravídico-puerperal. Cabe lembrar, de acordo com a Lei do Exercício Profissional da Enfermagem, Decreto n 94406/ 87, o pré-natal de baixo risco na gravidez pode ser inteiramente acompanhado pela enfermeira $(0)^{(7)}$.

Além de incluir mais um elemento da equipe de saúde no atendimento direto às gestantes, o Ministério da Saúde (MS) estabeleceu critérios para avaliação da Qualidade da assistência, a saber: número de consultas (6 no mínimo); início do acompanhamento pré-natal o mais precoce possível (obrigatoriamente até a $14^{\mathrm{a}}$ semana de gestação); distribuição das consultas durante o prénatal (no mínimo uma consulta no $1^{\circ}$ trimestre, duas no $2^{\circ}$ trimestre, três no $3^{\circ}$ trimestre e uma até 42 dias do puerpério); solicitação de exames complementares obrigatórios: hemograma; tipagem sangüínea e determinação do fator Rh; Coombs indireto (se necessário repetir na 24a $28^{\mathrm{a}}, 32^{\mathrm{a}}$ e $36^{\mathrm{a}}$ semana); urina I; teste anti-HIV; glicemia de jejum (na I a consulta, na $30^{\mathrm{a}}$ semana e devendo ser repetido no parto); sorologia para sífilis (na $1^{\circ}, 2^{\circ}$ e $3^{\circ}$ trimestre); realização do exame obstétrico durante o pré-natal (medida do peso, verificação pressão arterial, medida da aultura uterina, auscuta do batimento cardíaco fetal e toque vaginal Quando necessário); realização de atividades educativas (participação de, pelo menos, um grupo de sala de espera ou outra atividade correlata); orientação Quanto amamentação e parto; vacinação anti-tetânica (VAT); coleta de citopatológico de colo uterino ${ }^{(12,13)}$. No Que se refere aos exames laboratoriais salientamos Que outros autores recomendam a solicitação de outros exames: sorologia para toxoplamose IgM e IgG, sorologia para rubéola $\operatorname{lgM}$ e $\lg G$, sorologia para hepatite $B$, sorologia para hepatite $\mathrm{C}$, sorologia para citomegalovírus, exame parasitológico de fezes ${ }^{(6)}$.

O Ministério da Saúde preconizou também a avaliação permanente da assistência prestada, estabelecendo indicadores construídos a partir dos dados registrados nos prontuários das gestantes. Tal avaliação deve contemplar minimamente o Que se segue $\mathrm{e}^{(7)}$ :

- distribuição das gestantes por trimestre de início do pré-natal $\left(1^{\circ}, 2^{\circ}, 3^{\circ}\right)$;

- porcentagem de mulheres Que realizaram pré-natal em relação à população-alvo (número de gestantes na área ou previsto);

- porcentagem de abandono do pré-natal em relação ao total de mulheres inscritas;

- porcentagem de óbitos de mulheres, por causas associadas a gestação, parto ou puerpério, em relação ao total de gestantes atendidas;

- porcentagem de óbitos por causas perinatais, em relação ao total de recém-nascidos vivos;

- porcentagem de crianças com tétano neonatal, em relação ao total de recém-nascidos vivos;

- porcentagem de recém-nascidos vivos de baixo peso (menor de $2500 \mathrm{~g}$ ), em relação ao total de recém-nascidos vivos;

- porcentagem de VDRL positivos em gestantes e recémnascidos, em relação ao total de exames realizados;

- porcentagem de mulheres atendidas nos locais para onde foram referenciadas em relação ao total de mulheres Que retornaram à unidade de origem após encaminhamento.

Assim sendo, o pré-natal destaca-se como sendo o primeiro alvo a ser atingido euando se busca reduzir as altas taxas de morbimortalidade materna e perinatal e, para tanto, a viabilização dos programas exige atuação profissional competente.

Desta forma, este estudo teve como objetivo avaliar a Qualidade da assistência pré-natal prestada à gestantes de baixo risco, em uma Unidade de Saúde da Família, por meio da apreciação dos indicadores de Qualidade preconizados pelo Ministério da Saúde, acima mencionados.

\section{CASUÍSTICA E MÉTODO}

Trata-se de pesQuisa avaliativa, Quantitativa e retrospectiva realizada na Unidade de Saúde da Família (USF) de um município da Grande São Paulo.

O Programa de Saúde da Família (PSF), representa um mecanismo de organização de acesso (porta de entrada) a serviços simples e complexos (referência) e atualiza os conceitos tradicionais de distrito sanitário e a própria noção de hierarQuização da atenção à saúde observada no texto constitucional de 1988. Iniciado em 1994, o PSF apresentou um crescimento expressivo nos últimos anos. Dados do Ministério da Saúde de junho de 2006 afirmam Que 44,9\% da população brasileira está coberta pelo programa, sendo Que 5081 municípios estão se beneficiando dessa estratégia, somando-se a 25964 equipes de saúde da família em atuação no país ${ }^{(14)}$.

Como estratégia, o PSF tem os princípios operacionais de adscrição da clientela, da integralidade da atenção, do planejamento local e regional, da eqüidade do acesso aos serviços, do controle social, da ação intersetorial e do trabalho em equipe ${ }^{(15)}$.

Ressalta-se Que a assistência pré-natal prestada nas Unidades de Saúde da Família, no município onde este estudo foi realizado, é efetuada pelas enfermeiras e médicos e deve contemplar o preconizado pelo PHPN, seguindo as determinações da Secretaria Municipal de Saúde (SMS) no Que se refere aos critérios para o encaminhamento da gestante ao serviço de pré-natal de alto risco.

O levantamento dos dados foi feito por meio de análise dos prontuários das gestantes inscritas no programa de pré-natal da unidade, no período de março de 2003 a dezembro de 2004, Que iniciaram e terminaram o acompanhamento pré-natal, buscando identificar os indicadores preconizados pelo Ministério da Saúde ${ }^{(7)}$.

Vale salientar que esta pesquisa foi realizada após aprovação do Comitê de Ética em Pesquisa da Universidade de Mogi das Cruzes, instituição a Qual três das autoras encontravam-se vinculadas e pela Secretaria Municipal de Saúde (SMS), na Qual a Unidade de Saúde estudada encontra-se inserida.

\section{RESULTADOS E DISCUSSÃO}

Foram analisados 97 prontuários de gestantes cadastradas pelos 
Agentes Comunitários de Saúde (ACS) na área de abrangência da Unidade de Saúde da Família estudada. Dentre as gestantes cadastradas, $50(51,5 \%)$ realizaram pré-natal nesta unidade, 37 $(38,2 \%)$ realizaram o pré-natal em serviço particular e 10 (10,3\%) foram encaminhadas para o pré-natal de alto risco. Vale ressaltar Que os critérios analisados abaixo referem-se as gestantes Que realizaram pré-natal na Unidade Básica de Saúde em estudo.

No Que se refere a distribuição das gestantes por trimestre de início do pré-natal, $82 \%$ iniciaram o pré-natal no $1^{\circ}$ trimestre e $18 \%$ no $2^{\circ}$ trimestre. Evidenciando Que a cobertura do pré-natal foi adeQuada garantindo tanto o início precoce Quanto a realização de um número de consultas além do preconizado pela Organização Mundial de Saúde (OMS).

Os indicadores normalmente utilizados para avaliar a adeQuação da assistência pré-natal têm como parâmetro o número mínimo de consultas recomendado pelo American College of Ginecology and Obstetrics (ACOG), Que varia entre 11 e 14 consultas. No entanto, a OMS, mediante estudos Que demostraram Que não há diferenças significativas nos resultados perinatais com a redução do número de consultas durante o pré-natal, recomenda, no mínimo, 6 consultas pré-natais para uma gestação a termo, em gestantes sem fatores de riscos detectados, com o início precoce do acompanhamento, até o Quarto mês de gestação. Na população estudada o intervalo entre duas consultas não ultrapassaram 8 semanas, o Que também é recomendado pela $\mathrm{OMS}^{(8)}$.

O início precoce do acompanhamento pré-natal verificado neste estudo deve-se a busca ativa da gestante realizada pelas Agentes Comunitárias de Saúde (ACS), o Que confirma o apontado em outros estudos $^{(13)}$. Importante salientar Que o modelo adotado pelo PSF preconiza a delimitação da área de abrangência da unidade de saúde tornando possível detectar precocemente as gestantes residentes na comunidade e incentivá-las ao comparecimento no programa.

Quanto a porcentagem de mulheres Que realizaram pré-natal em relação à população-alvo; porcentagem de abandono do prénatal em relação ao total de mulheres inscritas e a porcentagem de óbitos de mulheres, por causas associadas à gestação, parto ou puerpério em relação ao total de gestantes atendidas encontrouse Que 100\% (50 mulheres) realizaram o pré-natal e não houve casos de abandono e/ou óbitos maternos, respectivamente. Estes achados apontam a importância do estabelecimento de mecanismos efetivos de regionalização do sistema local de saúde, como forma de Qualificar a assistência ${ }^{(16)}$.

Com relação a porcentagem de óbitos por causas perinatais, em relação ao total de recém-nascidos vivos e a porcentagem de crianças com tétano neonatal, em relação ao total de recém-nascidos vivos levantou-se Que não existiu QualQuer caso de óbitos por causas perinatais e/ou tétano neonatal.

A porcentagem de recém-nascidos vivos de baixo peso (menor de $2.500 \mathrm{~g}$ ), em relação ao total de recém-nascidos vivos foi de $2 \%$ (1 recém-nascido). Neste caso isolado o levantamento feito pelo prontuário da gestante evidenciou Que a mesma era tabagista consumindo aproximadamente 20 cigarros por dia. O Que coincide com os achados na literatura Que apontam o tabagismo como uma das principais causas do baixo peso ao nascer ${ }^{(6 ; 10)}$.

No Que se refere a porcentagem de VDRL positivos em gestantes e recém-nascidos, em relação ao total de exames realizados não houve resultados positivos, valendo ressaltar que do total de gestantes avaliadas Que iniciaram o pré-natal no $1^{\circ}$ trimestre, $82 \%$ (41 gestantes) realizaram 3 coletas do exame, eneuanto as Que iniciaram no $2^{\circ}$ trimestre (18\%) coletaram 2 amostras. Cabe lembrar da importância do início precoce do acompanhamento pré-natal viabilizando a realização de todos os exames preconizados ${ }^{(3,4,7)}$.

A porcentagem de mulheres (gestantes) atendidas nos locais para onde foram referenciadas em relação ao total de mulheres (puérperas) Que retornaram à unidade de origem após encaminhamentos foi de $88 \%$ (44 gestantes/puérperas). As puérperas Que não retornaram a unidade $12 \%$ (6 puérperas) não o fizeram pois mudaram da área de abrangência dado este obtido pela visita domiciliar realizada pelas ACS. De Qualquer forma, esses casos tiveram as consultas de puérperio agendadas por ocasião da notificação do nascimento do bebê, o que geralmente é feito pela própria puérpera ou familiares.

Mais uma vez enfatizamos a importância da busca ativa e dos registros em prontuário relacionados ao resultado do processo gestacional, ou seja, do acompanhamento puerperal, chamando a atenção para o fato de Que a proximidade geográfica facilita o contato dos usuários com a USF.

\section{CONSIDERAÇÕES FINAIS}

Inúmeros são os estudos Que tratam da avaliação da assistência pré-natal. Alguns contemplam os procedimentos mínimos aos Quais a mulher grávida deve ser submetida de forma Que se identifieue condições de risco à saúde materno-fetal, além da monitoração do estado de saúde do binômio e o tratamento de eventuais distúrbios. Entretanto, esta pesQuisa não avaliou a realização de tais procedimentos e sim indicadores de processo e resultados, o que propiciou evidenciar a adęuação de assistência pré-natal oferecida pela Unidade de Saúde estudada.

Os achados desta pesquisa permitiram destacar as especificidades do modelo de assistência preconizado pelo PSF, no Qual o cuidado é, usualmente, prestado pelos mesmos profissionais a cada oportunidade de contato do usuário com o serviço de saúde. Esta característica proporciona, no caso das gestantes, a segurança desejada e necessária no transcorrer da gravidez. No entanto, tal conforto ainda não é garantido por ocasião do parto, Quando constatase a dificuldade do acesso ao leito obstétrico e a falta de individualização do cuidado prestado.

Neste contexto, pode-se afirmar Que a oferta do cuidado individualizado aos usuários dos serviços de saúde pode representar uma motivação da sua preferência pelo modelo de assistência das Unidades do PSF e de outros serviços de atenção ao parto, como é o caso das Casas de Parto Normal.

A despeito da adequação da assistência pré-natal, constatada na Unidade pesquisada, observou-se algumas peculiaridades nos critérios de identificação do risco gestacional (Anexo I), o Qual difere do preconizado pelo Ministério da Saúde. Este achado é relevante, pois a eleição de critérios de diagnóstico de risco propicia a organização da oferta de recursos apropriados para cada caso específico.

Importante salientar Que, durante a realização deste estudo, nos mantivemos com a atenção voltada para a avaliação da Qualidade da assistência pré-natal e, ao finalizar a pesQuisa, acreditamos Que os resultados aQui apresentados poderão contribuir significativamente no sentido de subsidiar o cuidado à gestante, incrementar 
o ensino e a produção de conhecimento na área da Saúde da Mulher, bem como reforçar a importância da atuação de uma eQuipe multidisciplinar, dentro da Qual insere-se a Enfermeira, a Enfermeira
Obstétrica (o) e a Obstetriz (o) enquanto profissionais capazes de viabilizar e garantir a ampliação da cobertura da assistência prénatal no país e melhores resultados materno e perinatal.

\section{REFERÊNCIAS}

1. Ribeiro IM, Costa NR, Pinto LFS, Silva PLB. Atenção ao Prénatal na percepção das usuárias do Sistema Único de Saúde: um estudo comparativo. Cad Saúde Pública 2004; 20(2): 534-45.

2. Osis MIMD. Paism: um marco na abordagem da saúde reprodutiva no Brasil. Cad Saúde Públ 1998; 14 (supl. I): 25-32.

3. Ministério da Saúde (BR). Programa de Assistência Integral à Saúde da Mulher: Bases de Ação Programáticas. Brasília (DF): Ministério da Saúde; 1984.

4. Lopes GT, Portella VB, Lima ML, Penna LHG. Construindo uma proposta terapêutica de enfermagem no pré-natal de baixo risco. Esc Anna Nery Rev Enferm 2000; 4(1): 115 28.

5. Santos IS, Baloni RC, Minotto I, Klumb AG. Critérios de escolha de postos de saúde para o acompanhamento pré-natal em Pelotas, RS. Rev Saúde Pública 2000; 34 (6): 603-9.

6. Neme. B. Obstetrícia Básica. São Paulo (SP): Sarvier; 2000.

7. Ministério da Saúde (BR). Secretaria de Políticas de Saúde. Assistência Pré-Natal: manual técnico. Braślia (DF): Ministério da Saúde; 2000.

8. Coimbra LC, Silva AAM, Mochel EG, Alves MTSSB, Ribeiro VS, Aragão VMF, et al. Fatores associados à inadeQuação do uso da assistência pré-natal. Rev Saúde Pública 2003; 37(4): 456-62

9. Puccini RF, Pedroso GC, Silva EMK, Araujo NS, Silva NN.
Equidade na atenção pré-natal e ao parto em área da região metropolitana de São Paulo, 1996. Cad Saúde Pública 2003; 19(1):35-45.

10. Duarte MCA, Paula NMC, Acioly CMC. Perfil das usuárias de serviço de assistência pré-natal. Nursing 1999; 32-4.

11. Oba MDV, Tavares MSG. Aspectos positivos e negativos da assistência pré-natal no município de Riberão Preto SP. Rev Latino- am enfermagem 2000; 8(2): I I-7.

12. Serruya SI, Cecatti JG, Lago TG. Programa de humanização no pré-natal e nascimento do Ministério da Saúde no Brasil: resultados iniciais. Cad Saúde Pública 2004; 20(5): 1281-89.

13. Trevisan MR, Lorenzi DRS, Araújo NM, Ésber, K. Perfil da assistência pré-natal entre as usuárias do Sistema Único de Saúde em Caxias do Sul. RBGO 2002; 24(5): 293-9.

14. Ministério da Saúde (BR). Atenção Básica e a Saúde da Família; 2004. [citado em: 15 jun 2006]. Disponível em: URL: http:// portal.saude.gov.br/saude/

15. Peduzzi M. A inserção do enfermeiro na equipe de saúde da família na perspectiva da promoção da saúde. In Anais do $1^{\circ}$ Seminário Estadual: o enfermeiro no programa de saúde da família de São Paulo; 2000 nov 9- I I; São Paulo (SP), Brasil. São Paulo (SP): Secretaria de Estado da Saúde, 2000. p. I - I I .

16. Dias C. Auditoria médica: programa de pré-natal em posto de saúde na região Sul do Brasil. Rev Saúde Pública 2000; 34(4): 329-36. 